\title{
Comparison of Mathematical Approximation Methods for Mine Ventilation Network Analysis
}

\author{
Farhang Sereshki \\ Associate Professor \\ Faculty of Mining Engineering \\ Petroleum and Geophysics \\ Shahrood University of Technology \\ Shahrood, Iran \\ farhang@shahroodut.ac.ir
}

\author{
Amir Saffari \\ Ph.D Candidate \\ Faculty of Mining Engineering \\ Petroleum and Geophysics \\ Shahrood University of Technology \\ Shahrood, Iran \\ amirsaffari5710@yahoo.com
}

Ebrahim Elahi

Faculty of Engineering

Department of Mining Engineering

University of Sistan \& Baluchestan, Zahedan, Iran

elahi@eng.usb.ac.ir

\begin{abstract}
Various manual and computerized methods for analysis of mine ventilation network are presented. The selection of a suitable method depends on the target of network analysis. If the purpose of analysis is investigation of the effect of fans on mine network, using computerized methods based on mathematical approximation methods is better. The most notable feature has been the development of computerised mine ventilation network analysis. This has made prediction of fan requirements and airflow distributions in complex mine ventilation circuits feasible using desktop computers. A number of methods of mathematical approximations are presented such as the Hardy Cross method and its modified versions, Newton-Raphson technique, critical path, linear analysis, non-linear programming and optimization techniques. Using of Hardy Cross method in ventilation software has become common. In recent years, the use of Newton-Raphson technique in mine ventilation network analysis has been growing. Accordingly, in this paper, a model is developed for faster convergence to the final solution based on the Hardy Cross method that is named "conflation model of Hardy Cross method". Also, a more precise use of the Newton-Raphson technique in mine ventilation network analysis is also performed.
\end{abstract}

Keywords: Network Analysis, Mines Ventilation, Fans, optimization, Mathematical Approximations Methods.

\section{INTRODUCTION}

The basic requirement for the mine ventilation system is to provide air for people to breath and in a condition that will not cause any immediate or future ill effects. Because of the processes of mining, if positive airflow through the workings was not provided the air would very quickly become stale, contaminated and unfit for human consumption. The ventilation system must therefore be sufficient to deal with the contaminants released during mining. If they are not adequately dealt with as they are identified, they may become at best a discomfort to mine workers, and at worst cause serious or even fatal illness.

Although the mine environment by tunnel, winze and shaft is connected to the surface, this connection alone is not adequate for providing good airflow in the networks. It is necessary to ensure the air is clean by using various instruments and artificial methods. Accordingly, for dilution of harmful gases in the mine ventilation networks requires knowledge of the natural and artificial ventilation circuits (Elahi, 2014; Madani, 2006).

Ventilation design methods in underground mines are based on principles such as mapping, identification of ventilation branches and nodes, calculation of ventilation resistance of the each branches, the airflow intensity of the each branch, modified flow intensity, pressure loss for each branch, natural ventilation, network regularization and finally selection of the regulator doors or underground fans, along with selection main fan. 
Manual and computerized methods for the analysis of mine ventilation network are presented. The selection of the type of method depends on the target of network analysis. If the branch flow intensity and direction in a mine network have been fixed and determined in this state, using of manual methods for the analysis of the network is better.

The purpose of the mine ventilation network analysis in manual methods is only for the selection of main and auxiliary fans and the air regulators. But if the purpose of mine ventilation network analysis is the investigation of the effect of one or more fans on a mine network, use of mathematical approximation methods is better, because the manual method cannot calculate flow intensity in each of branches in complex networks.

The mathematical approximation methods begin with an assumption of flow intensity and direction in each of the branches of the mine network. The calculation error for each loop is determined using mathematical approximation equations, and then the assumed airflow intensity will be corrected. Airflow intensity corrections based on the provided mathematical equation continue to be conducted until calculation error is less than or equal to a required accuracy. Use of mathematical approximation methods for solving huge and complex networks by human is impossible; therefore utilisation of computers is necessary to solve them. A number of computer software's is available for analysis of mines ventilation networks. One of the most popular of them is Ventsim software that is based on Hardy Cross equation (Elahi, 2014; Madani, 2003). The first mathematical equations for estimating the flow intensity error in each loop, was presented by Hardy Cross in 1936. Although this equation was proposed for the analysis of water networks, this equation was later used and improved for mine ventilation network analysis by Wang (1982a) and El-Nagdy (2008). In addition to the mentioned methods, other techniques such as, Newton-Raphson technique (Madani \& Maleki, 2007; Wang, 1989), critical path (Wang, 1982b), linear analysis (Bhamidipati \& Procarione, 1985; Kamba et al., 1995), non-linear programming (Hu \& Longson, 1990; Wang, 1984) and optimization techniques (Collins, 1978) are also presented.

\section{A REVieW of APPRoximate Mathematics Methods}

\subsection{Hardy- Cross Method}

Hardy Cross's famous equation is as follows and according to equation 1, can be used to estimate the flow intensity error in each loop (Cross, 1936; Elahi, 2014).

$$
\begin{aligned}
& \left\{\begin{array}{l}
Q=Q_{0}+\Delta \\
\Delta P=R Q^{n}
\end{array} \rightarrow R Q^{n}=R\left(Q_{0}+\Delta\right)^{n} \rightarrow\right. \\
& R Q^{n}=R\left(Q_{0}{ }^{n}+n \Delta Q_{0}{ }^{n-1}+\cdots\right) \cong R Q_{0}{ }^{n}+n R \Delta Q_{0}{ }^{n-1} \\
& \rightarrow \sum R Q^{n}=\sum R Q_{0}{ }^{n}+\Delta \sum n R Q_{0}{ }^{n-1} \\
& \rightarrow\left\{\begin{array}{l}
\sum R Q^{n}=0 \\
\sum R Q_{0}^{n}=-\Delta \sum n R Q_{0}^{n-1}
\end{array}\right. \\
& \rightarrow \Delta=-\frac{\sum R Q_{0}{ }^{n}}{\sum n R Q_{0}{ }^{n-1}} \stackrel{n=2}{\longrightarrow} \Delta=-\frac{\sum R Q_{0}{ }^{2}}{2 \sum R Q_{0}} \\
& \rightarrow \Delta=-\frac{\sum \pm \Delta P_{i}}{2 \sum R_{i} Q_{i}}=-\frac{\sum \pm R_{i} Q_{i}^{2}}{2 \sum R_{i} Q_{i}}
\end{aligned}
$$

Where:

$Q:$ The actual flow intensity $\left(\mathrm{m}^{3} / \mathrm{sec}\right)$

$Q_{0}$ : The primary or assumed flow intensity $\left(\mathrm{m}^{3} / \mathrm{sec}\right)$

$\Delta:$ The flow intensity error in loop $\left(\mathrm{m}^{3} / \mathrm{sec}\right)$ 
$R:$ The mining work resistance in each branch (K Murgue)

$\Delta P:$ The pressure loss for each branch (mm water)

The Hardy- Cross equation solution steps are as follows:

\subsubsection{First Stage}

According to the junction law of equal flow into and out of every junction, assumed flow intensity with assumed direction for each of the branches of the mine ventilation network is established.

\subsubsection{Second Stage}

Identify closed loops in mine ventilation network according to equation (2) and select an assumed direction for them.

$$
N_{R}=N_{B}-N_{J}+1
$$

Where:

$N_{R}$ : Number of useful loops (with a surface node)

$N_{B}$ : Number of branches in mine ventilation network

$N_{J}:$ Number of junctions in mine ventilation network

\subsubsection{Third Stage}

Calculation of air pressure loss for each of branches in loop according to equation (3):

$$
\Delta P=R Q^{2}
$$

\subsubsection{Fourth Stage}

The calculation of flow intensity error for each loop according to equation (1): It should be noted for the numerator part of the equation, if the airflow direction in branch was aligned with the loop flow direction, the pressure loss is a positive sign, otherwise it is negative. For the denominator part of the equation it is always summed as a positive sign.

\subsubsection{Fifth Stage}

The calculation of the new flow for each branch of mine ventilation network: The error correction for each branch is determined by the algebraic summation of flow intensity error corrections of the loops contained the branch. It should be noted, if the loop direction was aligned with the branch flow direction, the error is applied as position; otherwise it is subtracted from the assumed flow. Also, if the value of new flow intensity in the branch is negative, the airflow direction in the branch is reversed.

\subsubsection{Sixth Stage}

Repeat the above operations from third to fifth stages until the calculation error is less than or equal to the required accuracy.

\subsection{Wang Modified Method}

Wang's correction is relating to fourth stage of the Hardy-Cross method. At this stage, the amount of flow intensity error of each loop is estimated according to equation (4). The purpose of this modification is that it eliminates the positive or negative sign of the pressure loss in equation (1), and also removes the decision to change the airflow direction if a flow is negative. Accordingly, if the flow direction changes, the amount of flow intensity changes to a positive sign, otherwise a negative sign should be appear in equation (4). In fact, the equations (1) and (4) for the flow intensity with positive value are quite similar.

$\Delta=-\frac{\sum_{i=1}^{i=n} b_{k i}\left(R_{i}\left|Q_{i}\right| Q_{i}-P_{n i}-P_{F i}\right)}{2 \sum_{1}^{i=n} b_{k i}^{2} R_{i}\left|Q_{i}\right|}$ 
Where:

$b_{k i}$ : Fundamental matrix element of the loop. If the airflow direction in branch was aligned with the loop flow direction, in this case, this element is equivalent to 1 in otherwise equivalent to -1 which appearance in equation (4).

$P_{n i}$ : Increasing pressure by reason of natural ventilation in the branch.

$P_{F i}$ : Increasing pressure by reason of installed fan in the branch.

\subsection{Newton- Raphson technique}

One of methods to solving numerical computation is using the Newton- Raphson technique. This technique is based on the definition of the derivative and the correction of it. In this technique, the initial guess of error value for the solution of the equation is estimated and then iteratively corrected. The mathematical equation can be expressed as follows:

$$
\begin{aligned}
& f(x)=0 \rightarrow f^{\prime}\left(x_{1}\right)=\frac{f\left(x_{2}\right)-f\left(x_{1}\right)}{x_{2}-x_{1}} \\
& \rightarrow x_{2}=x_{1}-\frac{f\left(x_{1}\right)}{f^{\prime}\left(x_{1}\right)}
\end{aligned}
$$

Where:

$x_{1}$ : Initial guess.

$x_{2}$ : Answer of the next step.

$f\left(x_{1}\right)$ : The value of the function on the basis of initial guess.

$f\left(x_{2}\right)$ : The value of the function on the basis of finally answer (is equivalent to 0 ).

$f^{\prime}\left(x_{1}\right)$ : The value of the derivative of function.

This technique for the first time was presented for the analysis of mine ventilation networks by Wang and $\mathrm{Li}$ in 1985. Also, this technique was used by Madani and Maleki in 2008 for $\Delta Q, H=\Delta P$ equations analysis in mine ventilation networks. Based on $\Delta Q, \Delta P=H$ equations analysis in mine ventilation networks by using Newton-Raphson technique is expressed as follows:

$x_{2}=x_{1}-\frac{f\left(x_{1}\right)}{f^{\prime}\left(x_{1}\right)} \rightarrow\left\{\begin{array}{l}\Delta Q_{n+1}=\Delta Q_{n}-\frac{f\left(H_{n}\right)}{\frac{\partial f_{n}}{\partial \Delta Q}} \\ H_{n+1}=H_{n}-\frac{f\left(H_{n}\right)}{\frac{\partial f_{n}}{\partial H}}\end{array}\right.$

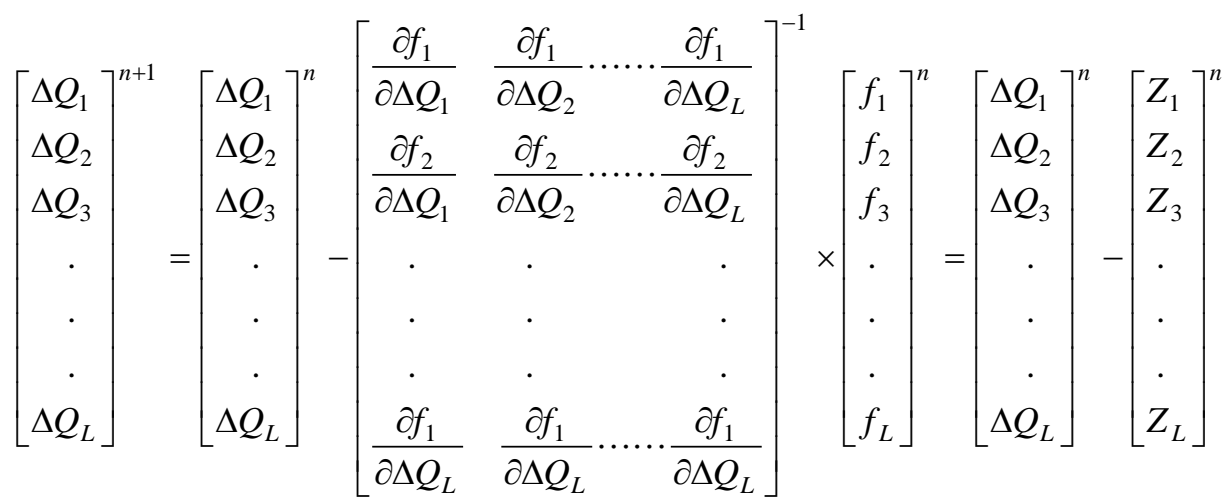


$\rightarrow\left[\begin{array}{ccc}\frac{\partial f_{1}}{\partial \Delta Q_{1}} & \frac{\partial f_{1}}{\partial \Delta Q_{2}} \cdots \cdots \cdot \frac{\partial f_{1}}{\partial \Delta Q_{L}} \\ \frac{\partial f_{2}}{\partial \Delta Q_{1}} & \frac{\partial f_{2}}{\partial \Delta Q_{2}} \cdots \cdots \frac{\partial f_{2}}{\partial \Delta Q_{L}} \\ \cdot & \cdot \\ \cdot & \cdot \\ \cdot & \cdot \\ \frac{\partial f_{1}}{\partial \Delta Q_{L}} & \frac{\partial f_{1}}{\partial \Delta Q_{L}} \cdots \cdots \cdot \frac{\partial f_{1}}{\partial \Delta Q_{L}}\end{array}\right] \times\left[\begin{array}{c}Z_{1} \\ Z_{2} \\ Z_{3} \\ \cdot \\ \cdot \\ \cdot \\ Z_{L}\end{array}\right]=\left[\begin{array}{c}f_{1} \\ f_{2} \\ f_{3} \\ \cdot \\ \cdot \\ f_{L}\end{array}\right] \rightarrow\left[\begin{array}{c}\Delta Q_{1} \\ \Delta Q_{2} \\ \Delta Q_{3} \\ \cdot \\ \cdot \\ \cdot \\ \Delta Q_{L}\end{array}\right]^{n+1}=\left[\begin{array}{c}\Delta Q_{1} \\ \Delta Q_{2} \\ \Delta Q_{3} \\ \cdot \\ \cdot \\ \cdot \\ \Delta Q_{L}\end{array}\right]\left[\begin{array}{c}Z_{1} \\ Z_{2} \\ Z_{3} \\ \cdot \\ \cdot \\ Z_{L}\end{array}\right]$

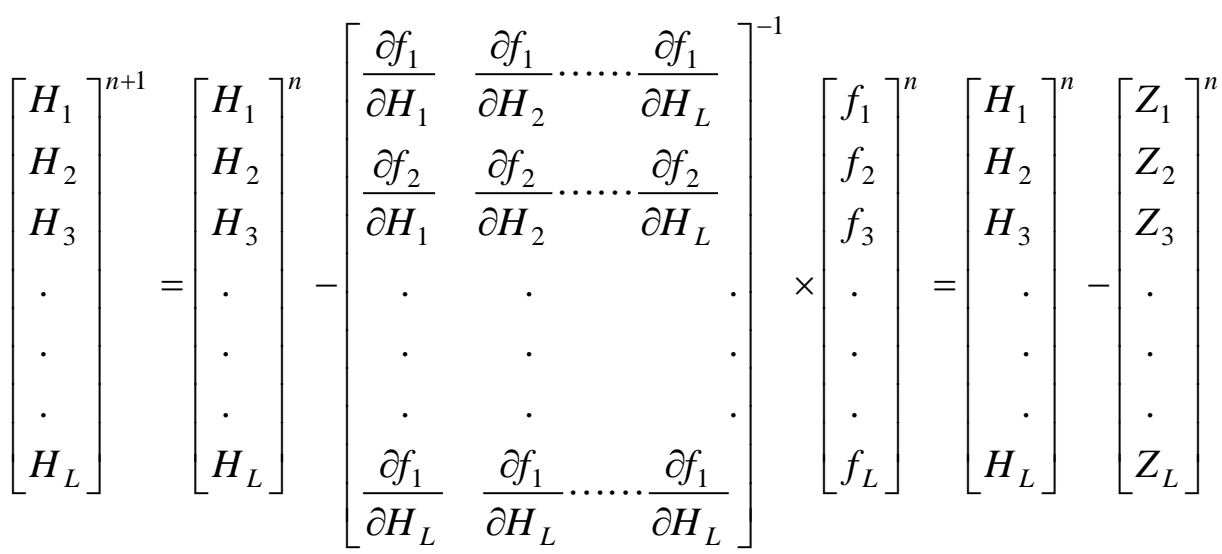

$\rightarrow\left[\begin{array}{cc}\frac{\partial f_{1}}{\partial H_{1}} & \frac{\partial f_{1}}{\partial H_{2}} \cdots \cdots \cdot \frac{\partial f_{1}}{\partial H_{L}} \\ \frac{\partial f_{2}}{\partial H_{1}} & \frac{\partial f_{2}}{\partial H_{2}} \cdots \cdots \frac{\partial f_{2}}{\partial H_{L}} \\ \cdot & \cdot \\ \cdot & \cdot \\ \frac{\partial f_{1}}{\partial H_{L}} & \frac{\partial f_{1}}{\partial H_{L}} \cdots \cdots \cdot \frac{\partial f_{1}}{\partial H_{L}}\end{array}\right] \times\left[\begin{array}{c}Z_{1} \\ Z_{2} \\ Z_{3} \\ \cdot \\ \cdot \\ Z_{L}\end{array}\right]=\left[\begin{array}{c}f_{1} \\ f_{2} \\ f_{3} \\ \cdot \\ \cdot \\ \cdot \\ f_{L}\end{array}\right] \rightarrow\left[\begin{array}{r}H_{1} \\ H_{2} \\ H_{3} \\ \cdot \\ \cdot \\ H_{L}\end{array}\right]^{n+1}=\left[\begin{array}{c}H_{1} \\ H_{2} \\ H_{3} \\ \cdot \\ \cdot \\ \cdot \\ H_{L}\end{array}\right]^{n}\left[\begin{array}{c}Z_{1} \\ Z_{2} \\ Z_{3} \\ \cdot \\ \cdot \\ Z_{L}\end{array}\right]$

The Newton-Raphson technique solution steps based on $\Delta Q$ equation are as follows:

\subsubsection{First stage}

According to branches' law, assumption flow intensity with assumption direction in each of the branches of the mine ventilation network is considered.

\subsubsection{Second stage:}

Identification of useful loops in mine ventilation network according to equation (2) and select an assumed direction for them.

\subsubsection{Third stage:}

Calculation of $\Delta Q$ equation based on air pressure loss for each branch in the network $\left(\Delta P=R Q^{2}\right)$.

\subsubsection{Fourth stage:}

Repeat the above operations from third stage until calculation error is less than or equal to the required accuracy. 


\section{Conflation Model of Hardy- Cross Method}

This correction is relating to fourth and fifth stages of the Hardy-Cross method. In the primary and modified methods, these two stages were calculated separately. Meaning that after completing the fourth stage calculations for all of the loop errors, the fifth stage of the operation to apply the loops errors to the branches begins. But if these two stages are applied to each loop together, the influence of each on each loop correction can be considered on others, and the investigation has led to more rapid access to the final answer. In other words, after the first loop error calculation is done, the relevant branches are corrected for flow intensity and only then are the second loop calculation error performed. Therefore, solving of fourth and fifth stages step by step together of Hardy-Cross method the investigation has led to more rapid access to the final answer. To prove this claim, consider the following two models.

\subsection{First Model}

An example mine's ventilation network, according to Figure 1 is considered by a fan with a pressure of 100 ( $\mathrm{mm}$ water). The mining resistance and flow intensity of each branch in this network is shown in Table 1. Initially, this network was simulated with Ventsim software that so far is the most complete mine ventilation network analysis software known, and then the results are compared with manual method to solving the Hardy- Cross equations, the Wang modified method and the conflation model. The result of simulation with Ventsim software for flow intensity distribution of each branch in the mentioned network is shown in Figure 2.

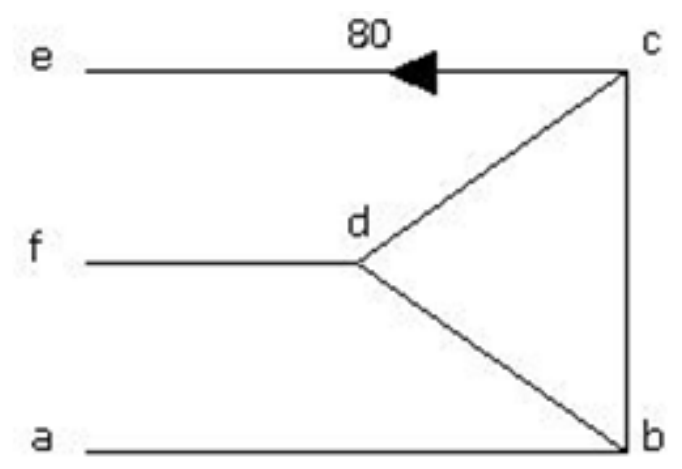

Figure1. Mine ventilation hypothetical network

Table1. Mine ventilation hypothetical network properties

\begin{tabular}{|c|c|c|}
\hline Branch & Mining resistance (k Murgue) & Flow intensity $\left(\mathrm{m}^{3} / \mathrm{sec}\right)$ \\
\hline$a b$ & 0.15 & 14 \\
\hline$b d$ & 0.14 & 4 \\
\hline$d f$ & 0.11 & 16 \\
\hline$b c$ & 0.12 & 18 \\
\hline$d c$ & 0.13 & 12 \\
\hline$c e$ & 0.10 & 30 \\
\hline
\end{tabular}

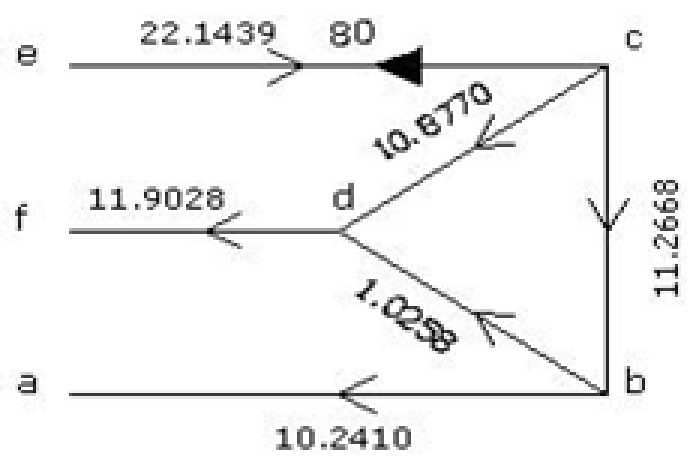

Figure2. Distribution of flow intensity with Ventsim software

For analysis of flow intensity of each branches by Hardy- Cross method, requires to assumption flow intensity. The assumed flows intensity with required loops selection is shown in Figure 3. 


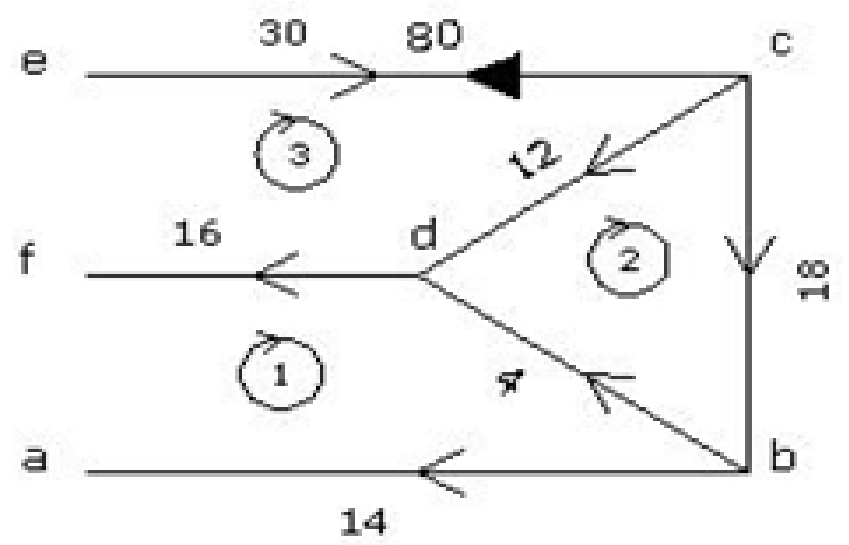

Figure3. Hypothetical flows intensity with loop selection

The first stage of calculation of Hardy- Cross method and Wang modified method (change the airflow direction where flow intensity has a negative value) is according to equation (1) and (2) which is as follows. Results of other stages are shown in Table 2.

$$
\begin{aligned}
& \Delta_{1}=-\frac{-0.11 \times 16^{2}-0.14 \times 4^{2}+0.15 \times 14^{2}}{2(0.11 \times 16+0.14 \times 4+0.15 \times 14)}=0.1131 \\
& \Delta_{2}=-\frac{0.14 \times 4^{2}-0.13 \times 12^{2}+0.12 \times 18^{2}}{2(0.14 \times 4+0.13 \times 12+0.12 \times 18)}=-2.6168 \\
& \Delta_{3}=-\frac{0.1 \times 30^{2}+0.13 \times 12^{2}+0.11 \times 16^{2}-80}{2(0.1 \times 30+0.13 \times 12+0.11 \times 16)}=-4.5 \\
& Q_{a b}=Q_{a b}+\Delta_{1}=14+0.1131=14.1131 \\
& Q_{b d}=Q_{b d}-\Delta_{1}+\Delta_{2}=4-0.1131+(-2.6168)=1.2701 \\
& Q_{d f}=Q_{d f}-\Delta_{1}+\Delta_{3}=16-0.1131+(-4.5)=11.3869 \\
& Q_{b c}=Q_{b c}+\Delta_{2}=18+(-2.6168)=15.3832 \\
& Q_{d c}=Q_{d c}-\Delta_{2}+\Delta_{3}=12-(-2.6168)+(-4.5)=10.1168 \\
& Q_{c e}=Q_{c e}+\Delta_{3}=30+(-4.5)=25.5
\end{aligned}
$$

According to Table 2 and after 23 iterations, the method reached conclusion with the results obtained from Ventsim software being quite similar. Therefore, the Wang modified method in this model is not able to converge faster to reach the final solution.

Table2. The results of Hardy Cross Method and its corrected stages

\begin{tabular}{|c|c|c|c|c|c|c|}
\hline Stage & $Q_{a b}$ & $Q_{b d}$ & $Q_{b c}$ & $Q_{c e}$ & $Q_{d f}$ & $Q_{d c}$ \\
\hline 1 & 14.1131 & 1.2701 & 15.3832 & 25.5 & 11.3869 & 10.1168 \\
\hline 2 & 11.9441 & 1.1454 & 13.0895 & 24.2697 & 12.3256 & 11.1802 \\
\hline 3 & 11.2632 & 1.1206 & 12.3838 & 23.1369 & 11.8737 & 10.7531 \\
\hline 4 & 10.7327 & 1.0679 & 11.8006 & 22.7312 & 11.9985 & 10.9306 \\
\hline 5 & 10.5244 & 1.0522 & 11.5766 & 22.4281 & 11.9038 & 10.8516 \\
\hline 10 & 10.2522 & 1.0268 & 11.2790 & 22.1567 & 11.9045 & 10.8776 \\
\hline 15 & 10.2415 & 1.0258 & 11.2674 & 22.1444 & 11.9029 & 10.8770 \\
\hline 20 & 10.2411 & 1.0258 & 11.2669 & 22.1439 & 11.9028 & 10.8770 \\
\hline 23 & 10.2410 & 1.0258 & 11.2668 & 22.1439 & 11.9028 & 10.8770 \\
\hline
\end{tabular}

The first stage of calculation of conflation model of Hardy-Cross method is as follows and results of other stages are shown in Table 3. 


$$
\begin{aligned}
& \Delta_{1}=-\frac{-0.11 \times 16^{2}-0.14 \times 4^{2}+0.15 \times 14^{2}}{2(0.11 \times 16+0.14 \times 4+0.15 \times 14)}=0.1131 \\
& Q_{a b}=Q_{a b}+\Delta_{1}=14+0.1131=14.1131 \\
& Q_{b d}=Q_{b d}-\Delta_{1}=4-0.1131=3.8869 \\
& Q_{d f}=Q_{d f}-\Delta_{1}=16-0.1131=15.8869 \\
& \Delta_{2}=-\frac{0.14 \times 3.8869^{2}-0.13 \times 12^{2}+0.12 \times 18^{2}}{2(0.14 \times 3.8869+0.13 \times 12+0.12 \times 18)}=-2.6119 \\
& Q_{b d}=Q_{b d}+\Delta_{2}=3.8869+(-2.6119)=1.2750 \\
& Q_{d c}=Q_{d c}-\Delta_{2}=12-(-2.6119)=14.6119 \\
& Q_{b c}=Q_{b c}+\Delta_{2}=18+(-2.6119)=15.3881 \\
& \Delta_{3}=-\frac{0.13 \times 14.6119^{2}+0.11 \times 15.8869^{2}+0.1 \times 30^{2}-80}{2(0.13 \times 14.6119+0.11 \times 15.8869+0.1 \times 30)}=-4.9284 \\
& Q_{d f}=Q_{d f}+\Delta_{3}=15.8869+(-4.9284)=10.9585 \\
& Q_{d c}=Q_{d c}+\Delta_{3}=14.6119+(-4.9284)=9.6835 \\
& Q_{c e}=Q_{c e}+\Delta_{3}=30+(-4.9284)=25.0716
\end{aligned}
$$

According to Table 3 and after 12 times iterations doing the method reached the conclusion with the results obtained from the Ventsim software being quite similar. Therefore, the conflation model of Hardy-Cross method is able to converge faster to reach the solution the final answer.

Table3. The results of conflation model of Hardy-Cross Method stages

\begin{tabular}{|c|c|c|c|c|c|c|}
\hline Stage & $Q_{a b}$ & $Q_{b d}$ & $Q_{b c}$ & $Q_{c e}$ & $Q_{d f}$ & $Q_{d c}$ \\
\hline 1 & 14.1131 & 1.2750 & 15.3881 & 25.0716 & 10.9585 & 9.6835 \\
\hline 2 & 11.7652 & 1.1230 & 12.8882 & 23.1244 & 11.3592 & 10.2363 \\
\hline 3 & 10.7573 & 1.0371 & 11.7944 & 22.4456 & 11.6883 & 10.6512 \\
\hline 4 & 10.3994 & 1.0249 & 11.4243 & 22.2323 & 11.8330 & 10.8080 \\
\hline 5 & 10.2874 & 1.0250 & 11.3124 & 22.1693 & 11.8819 & 10.8570 \\
\hline 10 & 10.2411 & 1.0258 & 11.2669 & 22.1439 & 11.9028 & 10.8770 \\
\hline 12 & 10.2410 & 1.0258 & 11.2668 & 22.1439 & 11.9028 & 10.8770 \\
\hline
\end{tabular}

\subsection{Second Model}

An assumed mine's ventilation network, according to Figure 4 is considered with two fans with the 100 and 80 ( $\mathrm{mm}$ water) pressures respectively. The mining resistance and flow intensity of each branch in this network is shown in Table 1. Initially, this network was simulated with Ventsim software, and then the results are compared with the manual method of solving the Hardy Cross equations, the Wang modified method and the conflation model. The result of simulation with Ventsim software for flow intensity distribution of each branch is shown in Figure 5.

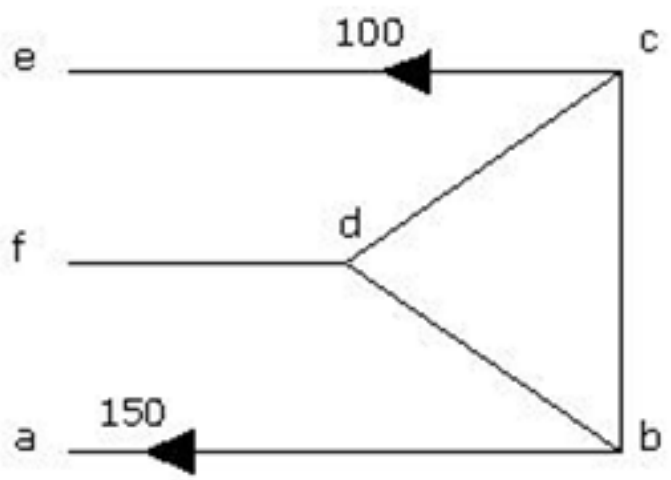

Figure4. Mine ventilation hypothetical network 


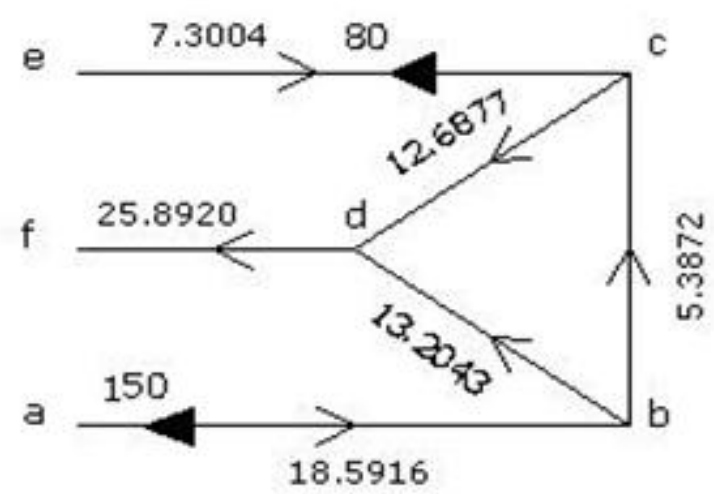

Figure5. Distribution of flow intensity with Ventsim software

For analysis of flow intensity of each branches by Hardy- Cross method, requires to assumption flow intensity. These assumption flows intensity with required loops selection is shown in Figure 6.

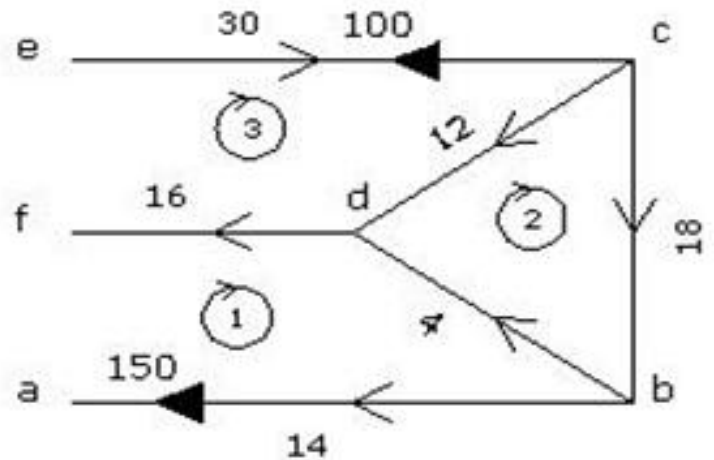

Figure6. Hypothetical flows intensity with loop selection

The first stage calculation of the Hardy- Cross method and the Wang modified method (change the airflow direction where flow intensity is a negative value) is according to Table 4. After 54 times iterations the results compared with the Ventsim software are quite similar. Therefore, the Wang modified method in this model is not able to converge faster to reach the final answer solution.

Table4. The results of Hardy Cross Method and its corrected stages

\begin{tabular}{|c|c|c|c|c|c|c|}
\hline Stage & $Q_{a b}$ & $Q_{b d}$ & $Q_{b c}$ & $Q_{c e}$ & $Q_{d f}$ & $Q_{d c}$ \\
\hline 1 & -2.8552 & 18.2384 & 15.3832 & 27.0823 & 29.9375 & 11.6991 \\
\hline 2 & 3.1437 & 13.6982 & 10.5545 & 21.1182 & 24.2619 & 10.5637 \\
\hline 3 & 8.8274 & 16.6249 & 7.7975 & 19.1800 & 28.0074 & 11.3825 \\
\hline 4 & 9.8175 & 14.5423 & 4.7248 & 16.0994 & 25.9169 & 11.3746 \\
\hline 7 & 14.6612 & 14.2574 & -0.4037 & 11.9361 & 26.5973 & 12.3399 \\
\hline 10 & 16.7412 & 13.6145 & 3.1267 & 9.4873 & 26.2285 & 12.6140 \\
\hline 20 & 18.4633 & 13.2324 & 5.2310 & 7.4562 & 25.9195 & 12.6871 \\
\hline 30 & 18.5829 & 13.2062 & 5.3767 & 7.3110 & 25.8939 & 12.6876 \\
\hline 40 & 18.5910 & 13.2045 & 5.3865 & 7.3011 & 25.8921 & 12.6877 \\
\hline 50 & 18.5915 & 13.2043 & 5.3872 & 7.3005 & 25.8920 & 12.6877 \\
\hline 54 & 18.5916 & 13.2043 & 5.3872 & 7.3004 & 25.8920 & 12.6877 \\
\hline
\end{tabular}

The first stage of calculation of the conflation model of Hardy-Cross method is as follows and results of other stages are shown in Table 5.

$$
\begin{aligned}
& \Delta_{1}=-\frac{-0.11 \times 16^{2}+0.15 \times 14^{2}-0.14 \times 4^{2}+150}{2(0.11 \times 16+0.15 \times 14+0.14 \times 4)}=-16.8552 \\
& Q_{a b}=Q_{a b}+\Delta_{1}=14+(-16.8552)=-2.8552 \\
& Q_{b d}=Q_{b d}-\Delta_{1}=4-(-16.8552)=20.8552 \\
& Q_{d f}=Q_{d f}-\Delta_{1}=16-(-16.8552)=32.8552
\end{aligned}
$$




$$
\begin{aligned}
& \Delta_{2}=-\frac{0.14 \times 20.8552^{2}-0.13 \times 12^{2}+0.12 \times 18^{2}}{2(0.14 \times 20.8552+0.13 \times 12+0.12 \times 18)}=-6.1035 \\
& Q_{b d}=Q_{b d}+\Delta_{2}=20.8552+(-6.1035)=14.7517 \\
& Q_{d c}=Q_{d c}-\Delta_{2}=12-(-6.1035)=18.1035 \\
& Q_{b c}=Q_{b c}+\Delta_{2}=18+(-6.1035)=11.8965 \\
& \Delta_{3}=-\frac{0.13 \times 18.1035^{2}+0.11 \times 32.8552^{2}+0.1 \times 30^{2}-100}{2(0.13 \times 18.1035+0.11 \times 32.8552+0.1 \times 30)}=-8.4386 \\
& Q_{d f}=Q_{d f}+\Delta_{3}=32.8552+(-8.4386)=24.4166 \\
& Q_{d c}=Q_{d c}+\Delta_{3}=18.1035+(-8.4386)=9.6649 \\
& Q_{c e}=Q_{c e}+\Delta_{3}=30+(-8.4386)=21.5614
\end{aligned}
$$

As shown in Table 5, after 29 times iterations the conclusion reached is similar to Ventsim software. Therefore, the conflation model of Hardy-Cross method is able to converge faster to reach the solution.

Table5. The results of conflation model of Hardy Cross Method stages

\begin{tabular}{|c|c|c|c|c|c|c|}
\hline Stage & $Q_{a b}$ & $Q_{b d}$ & $Q_{b c}$ & $Q_{c e}$ & $Q_{d f}$ & $Q_{d c}$ \\
\hline 1 & -2.8552 & 14.7517 & 11.8965 & 21.5614 & 24.4166 & 9.6649 \\
\hline 2 & 7.9459 & 14.3534 & 6.4075 & 16.6701 & 24.6159 & 10.2625 \\
\hline 3 & 11.7563 & 14.1362 & 2.3799 & 13.4210 & 25.1773 & 11.0412 \\
\hline 4 & 14.1798 & 13.6850 & -0.4947 & 11.2403 & 25.4201 & 11.7351 \\
\hline 5 & 15.8277 & 13.3255 & 2.5022 & 9.7304 & 25.5581 & 12.2327 \\
\hline 10 & 18.3870 & 13.2122 & 5.1749 & 7.4790 & 25.8661 & 12.6539 \\
\hline 15 & 18.5772 & 13.2049 & 5.3723 & 7.3130 & 25.8901 & 12.6853 \\
\hline 20 & 18.5906 & 13.2044 & 5.3862 & 7.3013 & 25.8919 & 12.6875 \\
\hline 25 & 18.5915 & 13.2043 & 5.3872 & 7.3005 & 25.8920 & 12.6876 \\
\hline 29 & 18.5916 & 13.2043 & 5.3872 & 7.3004 & 25.8920 & 12.6877 \\
\hline
\end{tabular}

\section{Comparison between Conflation Model of Hardy-Cross Method AND NEWTON-RAPHSON TECHNIQUE}

So far, the comparison of the Newton- Raphson technique in the analysis of the mines ventilation network is not fully accurate. Therefore, for a more precise evaluation of this technique, a comparison was done between this model and conflation model of Hardy-Cross method, resulting in two different evaluation models.

\subsection{First Model}

An example mine ventilation network is considered according to Figure 1 and Table 1. According to Table 3, the conflation model of Hardy-Cross method is able to solve after 12 times iterations and the flow intensity is calculated as shown in Figure 2. The solving stages of calculation of NewtonRaphson technique in mines ventilation networks analysis according to Figure 3 and Table 6 are shown in 4 stages. Comparison of these results with the results obtained from the Ventsim software which as shown in Figure 2 is quite similar. 


$$
\begin{aligned}
& f_{1}=0.15\left(Q_{a b}+\Delta Q_{1}\right)^{2}-0.11\left(Q_{d f}-\Delta Q_{1}+\Delta Q_{3}\right)^{2}-0.14\left(Q_{b d}-\Delta Q_{1}+\Delta Q_{2}\right)^{2} \\
& \left\{f_{2}=0.14\left(Q_{b d}-\Delta Q_{1}+\Delta Q_{2}\right)^{2}-0.13\left(Q_{d c}-\Delta Q_{2}+\Delta Q_{3}\right)^{2}+0.12\left(Q_{b c}+\Delta Q_{2}\right)^{2}\right. \\
& f_{3}=0.11\left(Q_{d f}-\Delta Q_{1}+\Delta Q_{3}\right)^{2}+0.1\left(Q_{c e}+\Delta Q_{3}\right)^{2}+0.13\left(Q_{d c}-\Delta Q_{2}+\Delta Q_{3}\right)^{2}-80 \\
& \int \frac{\partial f_{1}}{\partial \Delta Q_{1}}=0.3\left(Q_{a b}+\Delta Q_{1}\right)+0.22\left(Q_{d f}-\Delta Q_{1}+\Delta Q_{3}\right)+0.28\left(Q_{b d}-\Delta Q_{1}+\Delta Q_{2}\right) \\
& \left\{\frac{\partial f_{1}}{\partial \Delta Q_{2}}=-0.28\left(Q_{b d}-\Delta Q_{1}+\Delta Q_{2}\right)\right. \\
& \frac{\partial f_{1}}{\partial \Delta Q_{3}}=-0.22\left(Q_{d f}-\Delta Q_{1}+\Delta Q_{3}\right) \\
& \frac{\partial f_{2}}{\partial \Delta Q_{1}}=-0.28\left(Q_{b d}-\Delta Q_{1}+\Delta Q_{2}\right) \\
& \left\{\frac{\partial f_{2}}{\partial \Delta Q_{2}}=0.28\left(Q_{b d}-\Delta Q_{1}+\Delta Q_{2}\right)+0.26\left(Q_{d c}-\Delta Q_{2}+\Delta Q_{3}\right)+0.24\left(Q_{b c}+\Delta Q_{2}\right)\right. \\
& \frac{\partial f_{2}}{\partial \Delta Q_{3}}=-0.26\left(Q_{d c}-\Delta Q_{2}+\Delta Q_{3}\right) \\
& \frac{\partial f_{3}}{\partial \Delta Q_{1}}=-0.22\left(Q_{d f}-\Delta Q_{1}+\Delta Q_{3}\right) \\
& \left\{\frac{\partial f_{3}}{\partial \Delta Q_{2}}=-0.26\left(Q_{d c}-\Delta Q_{2}+\Delta Q_{3}\right)\right. \\
& \frac{\partial f_{3}}{\partial \Delta Q_{3}}=0.22\left(Q_{d f}-\Delta Q_{1}+\Delta Q_{3}\right)+0.2\left(Q_{c e}+\Delta Q_{3}\right)+0.26\left(Q_{d c}-\Delta Q_{2}+\Delta Q_{3}\right) \\
& {\left[\begin{array}{ccc}
8.84 & -1.12 & -3.52 \\
-1.12 & 8.56 & -3.12 \\
-3.52 & -3.12 & 12.64
\end{array}\right]\left[\begin{array}{l}
Z_{1} \\
Z_{2} \\
Z_{3}
\end{array}\right]=\left[\begin{array}{l}
-1 \\
22.4 \\
56.88
\end{array}\right] \rightarrow\left\{\begin{array}{l}
Z_{1}=3.28397 \\
Z_{2}=5.51631 \\
Z_{3}=6.776144
\end{array} \rightarrow\left[\begin{array}{l}
\Delta Q_{1} \\
\Delta Q_{2} \\
\Delta Q_{3}
\end{array}\right]^{1}=\left[\begin{array}{l}
-3.28397 \\
-5.51631 \\
-6.77614
\end{array}\right]\right.} \\
& {\left[\begin{array}{rrr}
6.461475 & -0.49494 & -2.75172 \\
-0.49494 & 6.283473 & -2.79244 \\
-2.75172 & -2.79244 & 10.18894
\end{array}\right]\left[\begin{array}{l}
Z_{1} \\
Z_{2} \\
Z_{3}
\end{array}\right]=\left[\begin{array}{l}
-0.42148 \\
4.142896 \\
6.139427
\end{array}\right] \rightarrow\left\{\begin{array}{l}
Z_{1}=0.4701 \\
Z_{2}=1.16211 \\
Z_{3}=1.048013
\end{array} \rightarrow\left[\begin{array}{l}
\Delta Q_{1} \\
\Delta Q_{2} \\
\Delta Q_{3}
\end{array}\right]^{2}=\left[\begin{array}{l}
-3.75407 \\
-6.67842 \\
-7.82416
\end{array}\right]\right.} \\
& {\left[\begin{array}{ccc}
5.999542 & -0.30118 & -2.62458 \\
-0.30118 & 5.84047 & -2.82211 \\
-2.62458 & -2.82211 & 9.881858
\end{array}\right]\left[\begin{array}{l}
Z_{1} \\
Z_{2} \\
Z_{3}
\end{array}\right]=\left[\begin{array}{l}
-0.07063 \\
0.22741 \\
0.148264
\end{array}\right] \rightarrow\left\{\begin{array}{l}
Z_{1}=0.004927 \\
Z_{2}=0.054609 \\
Z_{3}=0.031908
\end{array}\left[\begin{array}{l}
\Delta Q_{1} \\
\Delta Q_{2} \\
\Delta Q_{3}
\end{array}\right]^{3}=\left[\begin{array}{l}
-3.759 \\
-6.73303 \\
-7.85606
\end{array}\right]\right.} \\
& {\left[\begin{array}{rrr}
5.978217 & -0.28727 & -2.61865 \\
-0.28727 & 5.819355 & -2.82801 \\
-2.61865 & -2.82801 & 9.875443
\end{array}\right]\left[\begin{array}{l}
Z_{1} \\
Z_{2} \\
Z_{3}
\end{array}\right]=\left[\begin{array}{l}
-0.00042 \\
0.000636 \\
0.000249
\end{array}\right] \rightarrow\left\{\begin{array}{l}
Z_{1}=-4.1 \mathrm{E}-05 \\
Z_{2}=0.000133 \\
Z_{3}=5.22 \mathrm{E}-05
\end{array} \rightarrow\left[\begin{array}{l}
\Delta Q_{1} \\
\Delta Q_{2} \\
\Delta Q_{3}
\end{array}\right]^{4}=\left[\begin{array}{l}
-3.75896 \\
-6.73316 \\
-7.85612
\end{array}\right]\right.}
\end{aligned}
$$

Table6. The results of Newton- Raphson Technique stages

\begin{tabular}{|c|c|c|c|c|c|c|}
\hline Stage & $Q_{a b}$ & $Q_{b d}$ & $Q_{b c}$ & $Q_{c e}$ & $Q_{d f}$ & $Q_{d c}$ \\
\hline 1 & 10.7160 & 1.7677 & 12.4837 & 23.2239 & 12.5078 & 10.7402 \\
\hline 2 & 10.2459 & 1.0756 & 11.3216 & 22.1758 & 11.9299 & 10.8543 \\
\hline 3 & 10.2410 & 1.0260 & 11.2670 & 22.1439 & 11.9029 & 10.8770 \\
\hline 4 & 10.2410 & 1.0258 & 11.2668 & 22.1439 & 11.9028 & 10.8770 \\
\hline
\end{tabular}




\subsection{Second Model}

An example mine ventilation network is considered according to Figure 4 and Table 1. According to Table 5, the conflation model of Hardy-Cross method is able to solve after 29 times iterations, the flow intensity is calculated as shown in Figure 5.

The solving stages of calculation of Newton-Raphson technique in mines ventilation networks analysis for this model is similar to first model, but the difference is the $\quad+150$ value added to $f_{1}$ function. Therefore, in accordance with Figure 6, the first to fourth stages of the calculations are shown as follows in Table 7. After the fourth stage the results of this technique will diverge and its results and results obtained from Ventsim software which has been presented in Figure 5 is not the same.

$$
\begin{aligned}
& {\left[\begin{array}{ccc}
8.84 & -1.12 & -3.52 \\
-1.12 & 8.56 & -3.12 \\
-3.52 & -3.12 & 12.64
\end{array}\right]\left[\begin{array}{l}
Z_{1} \\
Z_{2} \\
Z_{3}
\end{array}\right]=\left[\begin{array}{l}
149 \\
22.4 \\
36.88
\end{array}\right] \rightarrow\left\{\begin{array}{l}
Z_{1}=22.75768 \\
Z_{2}=9.854478 \\
Z_{3}=11.68774
\end{array}\left[\begin{array}{l}
\Delta Q_{1} \\
\Delta Q_{2} \\
\Delta Q_{3}
\end{array}\right]^{1}=\left[\begin{array}{l}
-22.7577 \\
-9.85448 \\
-11.6877
\end{array}\right]\right.} \\
& {\left[\begin{array}{lrr}
8.060979 & -4.7329 & -5.95539 \\
-4.7329 & 9.331173 & -2.64335 \\
-5.95539 & -2.64335 & 12.26119
\end{array}\right]\left[\begin{array}{l}
Z_{1} \\
Z_{2} \\
Z_{3}
\end{array}\right]=\left[\begin{array}{l}
40.89803 \\
34.52533 \\
27.57702
\end{array}\right] \rightarrow\left\{\begin{array}{l}
Z_{1}=79.20504 \\
Z_{2}=59.01308 \\
Z_{3}=53.44228
\end{array}\left[\begin{array}{l}
\Delta Q_{1} \\
\Delta Q_{2} \\
\Delta Q_{3}
\end{array}\right]^{2}=\left[\begin{array}{l}
-101.963 \\
-68.8676 \\
-65.13
\end{array}\right]\right.} \\
& {\left[\begin{array}{lll}
-4.37898 & -10.3866 & -11.6232 \\
-10.3866 & 2.270191 & -4.09176 \\
-11.6232 & -4.09176 & 8.688953
\end{array}\right]\left[\begin{array}{l}
Z_{1} \\
Z_{2} \\
Z_{3}
\end{array}\right]=\left[\begin{array}{l}
810.9264 \\
470.951 \\
362.6513
\end{array}\right] \rightarrow\left\{\begin{array}{l}
Z_{1}=-41.1124 \\
Z_{2}=-30.0618 \\
Z_{3}=-27.4154
\end{array}\left[\begin{array}{l}
\Delta Q_{1} \\
\Delta Q_{2} \\
\Delta Q_{3}
\end{array}\right]^{3}=\left[\begin{array}{l}
-60.8503 \\
-38.8058 \\
-37.7146
\end{array}\right]\right.} \\
& {\left[\begin{array}{rrr}
1.847253 & -7.29249 & -8.60987 \\
-7.29249 & 5.702813 & -3.40371 \\
-8.60987 & -3.40371 & 10.47066
\end{array}\right]\left[\begin{array}{l}
Z_{1} \\
Z_{2} \\
Z_{3}
\end{array}\right]=\left[\begin{array}{l}
215.8013 \\
124.6311 \\
96.70772
\end{array}\right] \rightarrow\left\{\begin{array}{l}
Z_{1}=-23.2938 \\
Z_{2}=-17.1868 \\
Z_{3}=-15.505
\end{array}\left[\begin{array}{l}
\Delta Q_{1} \\
\Delta Q_{2} \\
\Delta Q_{3}
\end{array}\right]^{4}=\left[\begin{array}{l}
-37.5565 \\
-21.6189 \\
-22.2095
\end{array}\right]\right.}
\end{aligned}
$$

Table7. The results of Newton-Raphson Technique stages

\begin{tabular}{|c|c|c|c|c|c|c|}
\hline Stage & $Q_{a b}$ & $Q_{b d}$ & $Q_{b c}$ & $Q_{c e}$ & $Q_{d f}$ & $Q_{d c}$ \\
\hline 1 & -8.7577 & 16.9032 & 8.1455 & 18.3123 & 27.0699 & 10.1667 \\
\hline 2 & -87.9627 & 37.0952 & -50.8676 & -35.1300 & 52.8327 & 15.7375 \\
\hline 3 & -46.8503 & 26.0446 & -20.8058 & -7.7146 & 39.1358 & 13.0912 \\
\hline 4 & -23.5565 & 19.9376 & -3.6189 & 7.7905 & 31.3470 & 11.4094 \\
\hline 5 & -2.4295 & 14.3553 & 11.9257 & 21.8638 & 24.2933 & 9.9381 \\
\hline 10 & -72.2536 & 32.7890 & -39.4646 & -24.6447 & 47.6089 & 14.8199 \\
\hline 15 & -61.7188 & 30.0079 & -31.7109 & -17.6276 & 44.0911 & 14.0833 \\
\hline 20 & 4.1949 & 12.6069 & 16.8017 & 26.2761 & 22.0812 & 9.4743 \\
\hline 25 & -0.0391 & 13.7246 & 13.6855 & 23.4559 & 23.4950 & 9.7704 \\
\hline 30 & -64.8736 & 30.8407 & -34.0329 & -19.7290 & 45.1446 & 14.3039 \\
\hline 35 & -10.1318 & 16.3891 & 6.2573 & 16.7334 & 26.8652 & 10.4761 \\
\hline 40 & -25.0300 & 20.3221 & -4.7078 & 6.8100 & 31.8400 & 11.5178 \\
\hline 45 & -8.8842 & 16.0597 & 7.1755 & 17.5644 & 26.4486 & 10.3889 \\
\hline 50 & 109.2174 & -15.1188 & 94.0987 & 96.2295 & -12.9880 & 2.1308 \\
\hline 55 & -19.6218 & 18.8944 & -0.7274 & 10.4123 & 30.0341 & 11.1397 \\
\hline
\end{tabular}

\section{Conclusions}

Various methods of manual and computerized analysis of mine ventilation network are presented. The selection of type of method depends on target of network analysis. If the purpose of mine ventilation network analysis is investigation of the effect of one or more fans on mine network, use of computerized methods based on mathematical approximation methods is better.

The Hardy Cross method for mathematical approximation methods for mines ventilation network analysis has become more common. The convergence of this method depends on values, initial assumed flows and direction and loops selection. In this method, if the value of new flow in the branch is negative, it must reverse the direction of the branch. 
Wang's correction is relating to fourth stage of the Hardy-Cross method. In this correction model, if the value of new flow intensity in branch is negative, in the direction of the branch stays the same, and the flow intensity becomes negative.

Conflation model of Hardy- Cross method is relating to fourth and fifth stages of the Hardy-Cross method. Accordingly, if these two stages are performed sequentially step by step together for each branch, and the influence of each on the other to be considered, in this case, the investigation has led to more rapid access to the final answer. In other words, after the first loop error calculation must be done to correct the relevant branches of flow intensity and then calculate the error of the second loop. According to the examples presented, conflation model is able to decrease the repeat of calculation of the Hardy-Cross method, approximately 50 percent.

Newton- Raphson technique is one of the mathematical approximation methods which, is using in mines ventilation network analysis in recent years, however, so far the exact validation is not performed. Based on the results of this study, it can be concluded that in some of the models, this technique is not able to calculate the real value of flow intensity in mines ventilation networks analysis.

If in a network, the assumed initial flows direction is done in accordance with the real flows direction, in this case, with full confidence it can be claimed that the most rapid way to reaching the final answer in the analysis of mines ventilation networks is using of Newton- Raphson technique.

If in a network, some of assumed flows directions are not be in accordance with the real flows direction, using of Newton- Raphson technique in the analysis of mines ventilation networks for reaching the final answer in some of models may result in divergence and incorrect answers.

\section{REFERENCES}

Bhamidipati, S. S., Procarione, J. A., 1985. Linear Analysis for the Solution of Flow Distribution Problems, Proceedings of the 2nd US Mine Ventilation Symposium, Mousset_Jones, P. (Ed.), Rotterdam, Netherlands, 645-654.

Collins, M., Cooper, L., Helgason, R., Kennington, J., LeBlanc, L., 1978. Solving the Pipe Network Analysis Problem Using Optimization Techniques, Management Science, Vol. 24, 747-760.

Cross, H., 1936. Analysis of Flow in Networks of Conduits or Conductors, Bulletin 286, Engineering Experiment Station, University of Illinois, Urbane.

Elahi, E., 2014. The Principles of Designing Ventilation in Mines, Publication of Jihad Amikabir University, Iran (In Persian).

El-Nagdy, K. A., 2008. Analysis of Complex Ventilation Networks in Multiple Fan Coal Mine, Ph.D thesis, West Virginia University.

Hu, W., Longson, I., 1990. The Optimization of Airflow Distribution in Ventilation Networks Using A Nonlinear Programming Method, Mining Science and Technology, Vol. 10, No. 2, 209-219.

Kamba, G. M., Jacques, E., Patigny, J., 1995. Application of the Simplex Method to the Optimal Adjustment of the Parameters of A Ventilation Network, Proceedingss of the 7th US Mine Ventilation Symposium, Wala, A. M. (Ed.), SME, Littleton, Co., 461-465.

Madani, H., 2003. Mines Ventilation, Vol. 2, Tehran: Amirkabir University of Technology (Tehran Polytechnic) Press, Iran (In Persian).

Madani, H., 2006. Mines Ventilation, Vol. 1, Print 5, Tehran: University Center Pub, Iran (In Persian).

Madani, H., Maleki, B., 2007. Mines ventilation network analysis using Newton- Raphson method based on DQ equations, Amirkabir Journal of Science \& Research, Vol. 66, 97-102, Iran (In Persian).

Madani, H., Maleki, B., 2008. Mines ventilation networks analysis based on $H$ equations in $Q$ Newton-Raphson method, Iranian Journal of Mining Engineering (IJME), Vol. 3, No. 5, 71-77.

Wang Y.J., Li S. 1985. The Newton Method of Calculating the Ventilation Network, Proceedings, 2nd Conference on the Use of Computers in the Coal Industry, AIME, New York, pp. 388-392.

Wang, Y. J., 1982(a). Ventilation Network Theory, Mine Ventilation and Air Conditioning, 2nd ed., H. L. Hartman (Ed.), Wiley-Interscience, NY, 167-195.

Wang, Y. J., 1982(b). Critical Path Approach to Mine Ventilation Networks with Controlled Flow, Trans. SME-AIME, Vol. 272, 1862-1872. 
Wang, Y. J., 1984. A Non-Linear Programming Formulation for Mine Ventilation Networks with Natural Splitting, International Journal of Rock Mechanics and Mining Science, Vol. 21, No. 1, $42-45$.

Wang, Y. J., 1989. A Procedure for Solving A More Generalized System of Mine Ventilation Network Equations, Proceedings of the 4th US. Mine Ventilation Symposium, SME, Littleton, Co., 419424. 